hep-th/0103220

SLAC-PUB-8797

\title{
Closed String Tachyons, AdS/CFT, and Large N QCD
}

\begin{abstract}
Allan Adams and Eva Silverstein
Institute for Theoretical Physics; University of California; Santa Barbara, CA 93106

Department of Physics and SLAC; Stanford University; Stanford, CA 94305/94309日
\end{abstract}

We find that tachyonic orbifold examples of AdS/CFT have corresponding instabilities at small radius, and can decay to more generic gauge theories. We do this by computing a destabilizing Coleman-Weinberg effective potential for twisted operators of the corresponding quiver gauge theories, generalizing calculations of Tseytlin and Zarembo and interpreting them in terms of the large- $\mathrm{N}$ behavior of twisted-sector modes. The dynamically generated potential involves double-trace operators, which affect large- $\mathrm{N}$ correlators involving twisted fields but not those involving only untwisted fields, in line with large-N inheritance arguments. We point out a simple reason that no such small radius instability exists in gauge theories arising from freely acting orbifolds, which are tachyon-free at large radius. When an instability is present, twisted gauge theory operators with the quantum numbers of the large-radius tachyons aquire VEVs, leaving a gauge theory with fewer degrees of freedom in the infrared, analogous to but less extreme than "decays to nothing" studied in other systems with broken supersymmetry. In some cases one is left with pure glue QCD plus decoupled matter and U(1) factors in the IR, which we thus conjecture is described by the corresponding (possibly strongly coupled) endpoint of tachyon condensation in the M/String-theory dual.

March 2001

1 allan, evas@slac.stanford.edu

*Work supported by DOE Contract DE-AC03-76SF00515. 


\section{Introduction and Summary}

Orbifold examples [1] [2] [3] provide one of the simplest testing grounds and applications of AdS/CFT duality [4] [5]. With less than maximal SUSY, the physics at low energies is less constrained, and new elements of the AdS/CFT dictionary emerge. One such element is the relation between the gravity-side cosmological term (dilaton potential) which typically gets generated in the absence of SUSY, and the finite-N beta functions and dimension spectrum of the gauge theory [1][6][7].

Another element that arises upon breaking supersymmetry is the possibility of a stringy tachyon in the twisted sector on the gravity side. This typically (in fact in all cases known to the authors) happens at large AdS radius when the orbifold is symmetric and fixes some or all of the points on the $S^{q}$ component of the gravity background.2 Freely-acting orbifolds on the $S^{q}$ have no twisted-sector tachyon at large radius, since the twisted-sector states must wind around the sphere and are therefore very heavy. In this paper, we find an interesting pattern in the corresponding instability structure of the small radius limit of these theories by investigating the dynamics of twisted operators in the appropriate weakly-coupled dual quiver gauge theories [8].

Building on work of Tseytlin and Zarembo [9], we study radiative corrections in $I I B /(-1)^{F}$ (Type 0) on $A d S_{5} \times S^{5}$ and more general non-supersymmetric non-freelyacting orbifolds by $\Gamma=Z_{n}$, identifying a Coleman-Weinberg effective potential [10] which leads to growth of the VEVs of certain twisted operators quadratic in the gauge theory scalars. This instability along a (partial) Coulomb branch of the quiver gauge theory describes D-branes splitting into fractional branes at the fixed point locus of the orbifold. This one-loop contribution to the effective potential involves double-trace operators which affect correlators involving twisted operators at leading order in $1 / N^{2}$ (corresponding to genus zero on the gravity side). It is the leading non-conformal effect at small 't Hooft coupling and introduces non-conformal correlators for twisted operators into the theory at large $N$.

This raises an interesting puzzle some of whose potential resolutions we discuss in $\S 3.2$. It has to do with the issue of whether and how the conformal symmetry of the gauge theory (or equivalently the $S O(4,2)$ isometry group of the $A d S_{5} \times S^{5} / Z_{n}$ ) should be preserved in correlation functions involving twisted operators in the QFT dual to the

2 A tachyon indeed appears in the twisted sector of the non-SUSY model studied in detail in [1]; the statement otherwise was in error. 
standard gravity-side orbifold construction of the $A d S_{5} \times S^{5}$ sigma model. As we will discuss in $\S 2$, there is a space of possible orbifold quantum field theories arising from the space of renormalization group trajectories consistent with the quantum symmetry and inheritance of untwisted operator correlation functions from the $\mathcal{N}=4$ theory. This suggests a corresponding space of dual gravity-side orbifold string backgrounds, generic elements of which may generalize the standard construction.

The one-loop calculation is reliable in this context for a large but finite range of nonzero VEVs for the scalar fields, including values near a local minimum of the effective potential. We find that a renormalization-group improved perturbation theory analysis using one-loop beta functions does not lead to any additional control for the calculation of the potential near the origin of the Coulomb branch. From this we conclude that the instability indicated by this potential is present but, depending on the form of the potential near the origin of the Coulomb branch, may be a global effect accessible only by tunneling.

The resulting instability, present in the large-N limit, drives the erstwhile tachyon (or at least a twisted state with the same quantum numbers as a large-radius tachyon) to condense. It is interesting to note that whatever the behavior near the origin, the instability is consistent with the reality of the operator dimensions observed in [11], though not with an uncorrected extrapolation of the Breitenlohner-Freedman bound to the regime of large curvatures and large RR field strengths.

For a freely-acting orbifold, the quiver gauge theory has no Coulomb branch, as the Dbranes are at the same codimension as the orbifold fixed point locus in spacetime and have no directions in which to split up into separated fractional branes. One correspondingly finds no instability in the effective potential for twisted operators; we therefore conjecture that the tachyon-freedom of this type of model persists to small radius. This constitutes an interesting prediction for the RR string theory in this limit, and a satisfying class of examples to contrast with the tachyonic models.

We also study a case with non-free orbifold action and discrete torsion which projects out the lowest-lying tachyon, leaving tachyonic modes with angular momentum along the sphere. At weak coupling we find no one-loop instability. However there is a Coulomb branch (again describing fractional D-branes) in this case, and we find no symmetries preventing instabilities from getting generated at higher order.

We then consider in more detail the effects of condensing the twisted modes in the unstable (non-freely-acting) cases. On the gravity side at large radius, condensing a tachyon is expected to drive the theory to a subcritical dimension (with compensating gradients 
for the dilaton and other gravity fields), since the zero-momentum tachyon is a relevant operator on the string worldsheet [12][13] [14]. One way in which this can happen is to lose and/or deform the dimensions corresponding to the $S^{5} / \Gamma$ and the radial direction in $A d S_{5}$. This suggests losing the scalars and perhaps non-chiral fermions on the gauge theory side, since these matter fields have quantum numbers associated to the sphere. Similarly, nonperturbative instabilities have been argued to drive the theory to an endpoint with a loss of degrees of freedom (decays to "nothing") [15].

We find on the field theory side that the Coleman-Weinberg potential indeed drives the theory toward one with fewer degrees of freedom at least in the IR: in some cases, pure glue $S U(N)$ QCD plus some decoupled matter and U(1) factors. This can be seen algebraically or from a simple geometric picture of the low-energy/near horizon limit of symmetrically distributed fractional D-branes. We conjecture that this pure glue QCD theory is described by the endpoint of tachyon condensation in the dual gravity theory, at least at long distance on the QFT side. As just discussed, this is likely to be a $D<10$ theory on the gravity side (which may or may not be a perturbative string theory, depending on the effective string coupling that emerges in the subcritical theory when the tachyon has become large and mixed with the dilaton and other string fields). This result is suggestive of Polyakov's proposal for QCD as a noncritical string [16]; our analysis suggests that if realized it could be connected to ordinary AdS/CFT by tachyon condensation. 3 More complicated models involving both open and closed strings on the gravity side may leave surviving quark flavors as well, but we leave this goal of getting full QCD for future work.

Of course there are other avenues toward the String/M dual of QCD, arising from the low-energy limit of relevant perturbations of the $\mathcal{N}=4$ theory as in for example [18. Our connection to non-SUSY AdS/CFT examples via tachyon condensation appears complementary to these. It involves a dynamical mechanism for eliminating the extra matter on the gauge theory side, but the starting point is a non-supersymmetric system less well-understood than the $\mathcal{N}=4$ theory. As with many applications of AdS/CFT, it can be taken as a lesson about the gravity side: the gauge theory remaining after condensation of the twisted operators gives a dual answer to the question of what happens to the closed string theory after tachyon condensation.

There have been interesting discussions on closed string tachyons in D-brane and/or AdS/CFT systems in [3] [11] [19] 20] 21]. The role of tachyons, tachyon condensation,

3 This possibility was suggested earlier in general terms by Minahan at the end of 17. 
and/or tachyon-freedom in other conjectured non-supersymmetric closed string dualities has been studied in other contexts previously for example in [22] [23] [6] [24][25] [14] [26]. Calculations making use of the relevance of open-string tachyon vertex operators appeared in for example [27]. It would be very interesting if these techniques could be transported back to closed-string theory to investigate further the hints of QCD emerging in the systems we study here. Indeed, there is some resemblance of the Coleman-Weinberg potentials we discuss here and formulas for an (open string) tachyon potential in [27].

It would be interesting to study potential relations of our results on instabilities on the gauge theory side to the kinds of gravitational instabilities studied in [15][28][29] and in particular in the AdS/CFT context in [30]. Aside from a few comments, we leave this for future work, and focus here on the field theory side at weak coupling.

The paper is organized as follows. In $\S 2$, we calculate the one-loop Coleman-Weinberg potential for a large class of $Z_{n}$ orbifold models with fixed points, exhibiting a nontrivial regime of validity of the one-loop analysis out on the classical Coulomb branch. In $\S 3$, we find two simple minima of the potential within this regime of validity at which the low-energy gauge theory contains pure glue QCD sectors. We study the N-dependence of our results from $\S 2$ and establish that at large $\mathrm{N}$ the dynamically generated potential affects correlators involving twisted operators, and does not affect untwisted correlators. We discuss a puzzle this raises and several possibilities which may lead to its resolution, and review the evidence we have gathered for the persistence of the tachyon to small radius in these examples. In $\S 4$ we turn to the case of freely-acting orbifolds (where there is no tachyon at large radius) and show that the branch along which such an instability would arise is absent in this case. In $\S 5$ we analyze an orbifold with discrete torsion which has no Coleman-Weinberg instability at one loop but does have a Coulomb branch along which one could emerge at higher orders. Finally in $\S 6$ we discuss future directions.

\section{2. $Z_{n}$ Orbifolds with Fixed Points and Effective Potential}

Given the duality between $\mathcal{N}=4 \mathrm{SYM}$ and IIB string theory on $A d S_{5} \times S^{5}$, we can obtain new dual pairs by orbifolding both sides of this "parent" duality by a discrete group $\Gamma \subset S O(6)$ [1] [2]. The gravity side is Type IIB string theory on $A d S_{5} \times S^{5} / \Gamma$, and the QFT side is a quiver gauge theory [8] obtained by taking the low-energy limit of the worldvolume 
theory on D3-branes at the corresponding $C^{3} / \Gamma$ orbifold singularity. $⿴$ On the gravity side, correlations of untwisted operators are inherited at genus zero. The corresponding planar diagrams, in particular those corresponding to the beta functions [1], of the quiver gauge theories are inherited from those of the parent $\mathcal{N}=4$ theory [31].5]

Twisted states in the orbifold string theory correpond to "twisted operators" of the orbifold gauge theory, which are gauge-invariant operators that do not descend from gaugeinvariant operators of the parent $\mathcal{N}=4$ theory. On the gravity side, non-freely-acting geometrical orbifolds have tachyons in the twisted sector at least at large radius, while freely-acting orbifolds do not have tachyons, since twisted-sector states are very massive; we will return to them in $\S 4$. The effective potential for twisted operators is not constrained by the $\mathcal{N}=4$ theory, so we should expect an effective potential for twisted operators at order $N^{2}$, whereas the effective potential for untwisted operators should appear only at order $N^{0}$; we will see this explicitly below.

We will find it instructive to study several different cases. In this section we will focus on non-freely-acting orbifolds, which fix some locus on the $S^{5}$. The most extreme case of this is the "Type 0 " theory [3], which is an orbifold by $(-1)^{F}$ which acts only on the spinors and thus fixes the entire spacetime [32]. The corresponding quiver theory has gauge group $S U(N)^{2}$ with six real adjoint scalars $X_{1}^{i}=\left(\mathbf{N}^{\mathbf{2}}-\mathbf{1}, \mathbf{1}\right), X_{2}^{i}=\left(\mathbf{1}, \mathbf{N}^{\mathbf{2}}-\mathbf{1}\right)$, $i=1, \ldots, 6$, four Weyl fermions $\chi^{\alpha}$ in the bifundamental representation $(\mathbf{N}, \overline{\mathbf{N}})$, and four Weyl fermions $\psi^{\alpha}$ in the $(\overline{\mathbf{N}}, \mathbf{N})$.

These representations arise from projections of a parent $S U(2 N) \mathcal{N}=4$ theory. In a convenient basis, the gauge fields and scalars sit in diagonal blocks of this $S U(2 N)$ theory and the fermions sit in the off-diagonal blocks. The tree-level interactions of the orbifold

4 We are working with orbifolds of the maximally supersymmetric version of the duality. The duality being a statement of equivalence between two descriptions of one and the same system, the orbifold (including its instabilities) tautologically exists on one side if it exists on the other. Note that this equivalence is between the quantum theories, which introduces subtleties having to do with the manifestation on the gravity side of the choice of renormalization condition in the gauge theory side as we will discuss shortly.

5 It is worth emphasizing that we here take the regular representation for the action $\gamma_{a b}$ of the orbifold group on the Chan-Paton indices, one where $\operatorname{tr} \gamma=0$. With any other choice of action, the D-branes are a source for the twisted-sector tachyon in the asymptotically flat region away from the core of the D-branes, which does not decouple from the near-horizon low energy D-brane theory [20]. 
theory are those of the $S U(2 N) \mathcal{N}=4$ theory which involve fields which survive the orbifold projection. In addition to the minimal gauge couplings, one has quartic scalar interactions of the $X_{1}^{i}$ which are identical to those of an $S U(N) \mathcal{N}=4$ theory and likewise for $X_{2}^{i}$. The bifundamental fermions mix the two $S U(N)$ gauge groups via tree-level Yukawa interactions of the form6

$$
g_{Y M}\left[\operatorname{tr}\left(\bar{\psi} X_{1} \psi\right)+\operatorname{tr}\left(\bar{\chi} \chi X_{1}\right)+\operatorname{tr}\left(\bar{\chi} X_{2} \chi\right)+\operatorname{tr}\left(\bar{\psi} \psi X_{2}\right)\right]
$$

The orbifold on the gravity side has a "quantum symmetry" $\Gamma$, [33], under which twisted states transform non-trivially. In the Type 0 case, this is a $Z_{2}$ symmetry which is manifested on the gauge theory side by a symmetry exchanging the two $S U(N)$ factors in the gauge group (and correspondingly exchanging $X_{1}^{i} \leftrightarrow X_{2}^{i}$ and $\psi \leftrightarrow \chi$ ). The lowestdimension twisted operators in our theory are thus $\operatorname{tr} X_{1}^{2}-\operatorname{tr} X_{2}^{2}$ and $\operatorname{tr} X_{1}^{i} X_{1}^{j}-\operatorname{tr} X_{2}^{i} X_{2}^{j}$, which have dimension two at leading order. In the full D-brane system, these operators couple to two derivatives of the tachyon field in the directions transverse to the D-branes [34], while the tachyon itself couples to the dimension four operator $\operatorname{tr}\left(F_{1}^{2}+D X_{1} D X_{1}-F_{2}^{2}-D X_{2} D X_{2}\right)$ [34] [1].

The interactions of untwisted states are inherited at genus zero, but those of twisted states are constrained a priori only by the quantum symmetry. Our main interest will be contributions to the scalar potential generated by quantum corrections to the gauge theory at leading order in the $1 / N^{2}$ expansion. We will discuss the calculation of the effective potential in non-freely-acting $Z_{n}$ orbifolds in the remainder of this section, and then interpret the results in terms of tachyons and explore the instability structure on the gauge theory side in $\S 3$.

In [9], Tseytlin and Zarembo calculated the bosonic potential energy lifting the classical moduli space of the Type 0 theory at one-loop order in the gauge theory. Because of the quartic scalar interactions, the classical moduli space of this theory is parameterized by the eigenvalues of commuting matrices $\left\langle X_{1}^{i}\right\rangle \equiv \operatorname{diag}\left(x_{1}^{i, 1}, \ldots, x_{1}^{i, N}\right)$ and $\left\langle X_{2}^{i}\right\rangle \equiv \operatorname{diag}\left(x_{2}^{i, 1}, \ldots, x_{2}^{i, N}\right)$ with $\Sigma_{a} x_{1}^{i, a}=0=\Sigma_{a} x_{2}^{i, a}$. Going to a generic point on this

6 In this section we henceforth assume canonical normalization for the kinetic terms with no factors of $1 / g_{Y M}^{2}$ in front of the action. 
moduli space and integrating out massive particles, one obtains a simple expression for the vacuum energy as a function of $x_{1}^{i, a}$ and $x_{2}^{i, a}[9]$ :

$$
\begin{aligned}
& V_{e f f} \sim \frac{g_{Y M}^{4}}{8 \pi^{2}} \Sigma_{a, b=1}^{N}\left[\left|x_{1}^{a}-x_{1}^{b}\right|^{4} \log \frac{\left|x_{1}^{a}-x_{1}^{b}\right|^{2}}{\tilde{M}^{2}}+\left|x_{2}^{a}-x_{2}^{b}\right|^{4} \log \frac{\left|x_{2}^{a}-x_{2}^{b}\right|^{2}}{\tilde{M}^{2}}\right. \\
& \left.-2\left|x_{1}^{a}-x_{2}^{b}\right|^{4} \log \frac{\left|x_{1}^{a}-x_{2}^{b}\right|^{2}}{\tilde{M}^{2}}\right]
\end{aligned}
$$

where $|x|^{2} \equiv x^{i} x^{i} \equiv \vec{x}^{2}$ and $\tilde{M}$ is related to a subtraction point to be discussed shortly. The first two terms in (2.2) arise from integrating out the off-diagonal entries in $\left(\vec{X}_{1}\right)_{a b},\left(A_{1}^{\mu}\right)_{a b}$ and $\left(\vec{X}_{2}\right)_{a b},\left(A_{2}^{\mu}\right)_{a b}$, which have masses $g_{Y M}\left|x_{1}^{a}-x_{1}^{b}\right|$ and $g_{Y M}\left|x_{2}^{a}-x_{2}^{b}\right|$ respectively. The last arises from integrating out the fermions $(\psi)_{a b}$ and $(\chi)_{a b}$ in the bifundamental $(\mathbf{N}, \overline{\mathbf{N}}) \oplus(\overline{\mathbf{N}}, \mathbf{N})$, whose masses are $g_{Y M}\left|x_{1}^{a}-x_{2}^{b}\right|$. This expression can be understood (and later generalized) as follows. Integrating out a particle of mass $m^{2}$ leads to a contribution

$$
(-1)^{F} \int d^{4} p \log \left(p^{2}+m^{2}\right) \sim(-1)^{F} \int_{0}^{\infty} \frac{d t}{t^{3}} e^{-t m^{2}}
$$

where $F$ is the spacetime fermion number and where we are ignoring coefficients of order one. This expression has quadratic and logarithmic divergences (as well as a quartic divergence in the vacuum energy to which the field theory is insensitive). We therefore require counterterms; following the analysis of [10] one obtains an expression of the form

$$
\begin{aligned}
& V_{e f f}=\Sigma_{a, b=1}^{N}\left[\left|x_{1}^{a}-x_{1}^{b}\right|^{4}\left(\lambda_{11}^{a b}+\left(A g_{Y M}^{4}+B\left(\lambda_{11}^{a b}\right)^{2}\right) \log \frac{\left|x_{1}^{a}-x_{1}^{b}\right|^{2}}{M^{2} e^{25 / 6}}\right)\right. \\
& +\left|x_{2}^{a}-x_{2}^{b}\right|^{4}\left(\lambda_{22}^{a b}+\left(A g_{Y M}^{4}+B\left(\lambda_{22}^{a b}\right)^{2}\right) \log \frac{\left|x_{2}^{a}-x_{2}^{b}\right|^{2}}{M^{2} e^{25 / 6}}\right) \\
& \left.-2\left|x_{1}^{a}-x_{2}^{b}\right|^{4}\left(\lambda_{12}^{a b}+\left(A g_{Y M}^{4}+B\left(\lambda_{12}^{a b}\right)^{2}\right) \log \frac{\left|x_{1}^{a}-x_{2}^{b}\right|^{2}}{M^{2} e^{25 / 6}}\right)\right]
\end{aligned}
$$

where $A$ and $B$ are constants of order 1 and $g_{Y M}$ and $\lambda_{i j}^{a b}$ are renormalized couplings. This potential includes the 1-loop contributions plus counterterms chosen to satisfy the renormalization conditions

$$
\left.\frac{d^{4} V_{e f f}}{d x_{i j}^{a b}{ }^{b}}\right|_{x_{i j}^{a b}=M}=\lambda_{i j}^{a b}
$$

where $x_{i j}^{a b}=x_{i}^{a}-x_{j}^{b}$. The coupling constants determined at one value of the subtraction point $M$ are related to those at a different point by the renormalization group. A choice of renormalization group trajectory is a choice of field theory and presumably corresponds to a 
choice of what the precise configuration of dual gravity-side string fields is. At small radius, we do not have an independent handle on the gravity side, so we will simply consider the whole set of possible trajectories consistent with the symmetries and inheritance properties of the orbifold. This issue will be discussed further in §3.2. As discussed in [9], there are planar contributions proportional to $N$ in the individual terms in the effective potential that must cancel in the orbifold theory by inheritance. This plus the quantum symmetry leads to the simplification that in the quantum field theory dual to the orbifold background, we should have $\lambda_{i j}^{a b} \equiv \lambda$.

As discussed in [9] (and as will be generalized and studied further in $§ 3$ ) there is an unstable direction in the potential in which $x_{1}^{1}=\rho=-x_{1}^{2}$, with all other $x_{i}^{a}=0$. Plugging this into (2.4), one finds

$$
V_{e f f} \sim \rho^{4}\left[\lambda+\left(A g_{Y M}^{4}+B \lambda^{2}\right) \log \frac{2^{8 / 3} \rho^{2}}{M^{2} e^{25 / 6}}\right]
$$

Let us now renormalize at a subtraction point of order the VEV of $\rho$, e.g. $M=2^{4 / 3}\langle\rho\rangle$, where

$$
\left.\frac{d V_{e f f}}{d \rho}\right|_{\rho=\langle\rho\rangle}=0
$$

Imposing this condition, one obtains in the theory expanded about the minimum of the potential a relation between $\lambda$ and $g_{Y M}$ as in [10] of the form

$$
\lambda=C g_{Y M}^{4}
$$

where $C$ is a constant of order 1 . So the renormalized quartic scalar coupling along the Coulomb branch is of order $g_{Y M}^{4}$, as befits a contribution at one-loop order in perturbation theory. Plugging this back into (2.4) and defining

$$
\tilde{M}^{2} \equiv e^{\frac{25}{6}-\frac{C}{A}} M^{2}
$$

we recover the result (2.2).

The one-loop result is reliable where the logarithms in (2.4) are not big enough to compensate the small couplings $\lambda, g_{Y M}$ and make different orders in perturbation theory commensurate. As in the original analysis of the massless Abelian Higgs model in [10], our result is reliable near minima of the potential but not at the origin $x_{i j}^{a b} \rightarrow 0$ or in the asymptotic region $x_{i j}^{a b} \rightarrow \infty$, since there the logarithms are large. In some theories, expressing the effective potential in terms of the running coupling (the solution of the 
Callan-Symanzik equations) results in a weakly coupled description for a larger range of $x$. As demonstrated below, this is not the case at one-loop order at large $\mathrm{N}$ in our theories, which have a somewhat remarkable RG structure due to the vanishing of untwisted beta functions at large N.8

The 1-loop $\beta$ functions are easily computed. For the Type 0 theory and the other quiver theories we are about to analyze, Large- $\mathrm{N}$ inheretance ensures that the gauge and yukawa couplings have vanishing $\beta$ functions at leading order in $\frac{1}{N}$ 31.

The $\beta$ function for the quartic scalar coupling $\left(\operatorname{tr} X_{1}^{2}-\operatorname{tr} X_{2}^{2}\right)^{2}$ can be calculated directly from our calculation of the renormalized coupling:

$$
\beta_{\lambda} \sim \lambda^{2}+g_{Y M}^{4}
$$

the resulting $\mathrm{RG}$ equations are solved by

$$
\lambda=g_{Y M}^{2} \operatorname{Tan}\left(g_{Y M}^{2} \ln \frac{\rho^{2}}{M^{2}}+g_{o}^{2}\right)
$$

As $\rho$ gets either very large or small compared to $M^{2} e^{\frac{\pi}{g_{Y M}^{2}}}$, this solution becomes strongly coupled and untrustworthy. So in these theories at one-loop order at large N, RG improvement does not help. While we can trust our one-loop effective potential near its local minimum, we cannot trust the dynamics near the origin, or at large values of the scalar VEV. Since the gauge coupling is protected from developing a $\beta$ function at large $N$ by inheritance, the main effect of higher loops will be to add higher monomials in $\lambda$, whose effects will depend strongly on their signs. We will leave this much more involved two-loop calculation to future work, and in this paper content ourselves with having identified at least a global instability. This leaves open the possibility that the region near $x \sim M$ could only be accessible via tunneling from the region of the origin. We will comment further on this in $\S 3$.

More generically one can consider locally-free orbifold actions. One example we will study in detail is a $C / Z_{3}$ orbifold, under which a single complex plane with coordinate $z^{1}$ is rotated by $\alpha \equiv e^{2 \pi / 3}: z^{1} \rightarrow \alpha^{2} z^{1}, z^{2,3} \rightarrow z^{2,3}$. This acts by a phase $\alpha^{ \pm 1} \equiv e^{ \pm 2 \pi / 3}$ on all the spacetime spinors, and so projects out all the massless gravitinos. The quiver theory

7 We thank D. Gross for interesting discussions on this. 
in this case has gauge group $S U(N)^{3}$. The matter content consists of four real scalars in the adjoint:

$$
\begin{array}{ll}
X_{1}^{i} & \left(\mathbf{N}^{\mathbf{2}}-\mathbf{1}, \mathbf{1}, \mathbf{1}\right) \\
X_{2}^{i} & \left(\mathbf{1}, \mathbf{N}^{2}-\mathbf{1}, \mathbf{1}\right) \\
X_{3}^{i} & \left(\mathbf{1}, \mathbf{1}, \mathbf{N}^{\mathbf{2}}-\mathbf{1}\right),
\end{array}
$$

for $i=1, \ldots, 4$; one complex scalar in the bifundamental representations:

$$
\begin{array}{ll}
U & (\mathbf{N}, \overline{\mathbf{N}}, \mathbf{1}) \\
V & (\mathbf{1}, \mathbf{N}, \overline{\mathbf{N}}) \\
W & (\overline{\mathbf{N}}, \mathbf{1}, \mathbf{N}),
\end{array}
$$

and four Weyl fermions in the bifundamental representations:

$$
\begin{array}{ll}
\chi_{U}^{\alpha} & (\mathbf{N}, \overline{\mathbf{N}}, \mathbf{1}) \\
\chi_{V}^{\alpha} & (\mathbf{1}, \mathbf{N}, \overline{\mathbf{N}}) \\
\chi_{W}^{\alpha} & (\overline{\mathbf{N}}, \mathbf{1}, \mathbf{N}),
\end{array}
$$

for $\alpha=1, \ldots, 4$. The interactions in this case are inherited from an $S U(3 N) \mathcal{N}=4$ theory. In a convenient basis, the gauge bosons and adjoint scalars sit in diagonal $N \times N$ blocks of the adjoint matrices of the parent theory, and the bifundamental scalars (2.13) and fermions (2.14) sit in off-diagonal blocks.

The Higgs branch of this gauge theory, along which $U=V=W$ (as enforced by the quartic scalar interactions inherited from the $\mathcal{N}=4$ D-terms) describes motion of the D3-branes away from the orbifold fixed locus. The theory also has a Coulomb branch, where $U=V=W=0$ and components of the $X_{k}^{i}$ for different $k$ get independent VEVs. This describes motion of "fractional" D-branes away from each other along the orbifold fixed locus $z^{1}=0$.

In this case, one finds an effective potential analogous to that of [9] (2.2):

$$
\begin{aligned}
& V_{e f f} \sim \frac{g_{Y M}^{4}}{8 \pi^{2}} \frac{3}{4} \Sigma_{a, b=1}^{N}\left[\left|x_{1}^{a}-x_{1}^{b}\right|^{4} \log \frac{\left|x_{1}^{a}-x_{1}^{b}\right|^{2}}{\tilde{M}^{2}}+\left|x_{2}^{a}-x_{2}^{b}\right|^{4} \log \frac{\left|x_{2}^{a}-x_{2}^{b}\right|^{2}}{\tilde{M}^{2}}\right. \\
& +\left|x_{3}^{a}-x_{3}^{b}\right|^{4} \log \frac{\left|x_{3}^{a}-x_{3}^{b}\right|^{2}}{\tilde{M}^{2}}-\left|x_{1}^{a}-x_{2}^{b}\right|^{4} \log \frac{\left|x_{1}^{a}-x_{2}^{b}\right|^{2}}{\tilde{M}^{2}} \\
& \left.-\left|x_{2}^{a}-x_{3}^{b}\right|^{4} \log \frac{\left|x_{2}^{a}-x_{3}^{b}\right|^{2}}{\tilde{M}^{2}}-\left|x_{3}^{a}-x_{1}^{b}\right|^{4} \log \frac{\left|x_{3}^{a}-x_{1}^{b}\right|^{2}}{\tilde{M}^{2}}\right]
\end{aligned}
$$


Here (similarly to the discussion following (2.2) ) the first three terms come from integrating out the four real scalars and the gauge fields which transform in the adjoint representation, which in this theory involves $3 / 4$ of the bosons in the theory, hence the factor of $3 / 4$ relative to the Type 0 result. The last three terms come from integrating out the bifundamental matter, which in this theory consists of one complex scalar (1/4 of the total bosons) and all of the fermions, leading to the factor of $-3 / 4$ appearing in front of these terms in (2.15).

It is now clear how to generalize this result to arbitrary $Z_{n}$ orbifolds. Consider for example a non-freely acting $Z_{n}$ orbifold with rotation angles $2 \pi\left(\frac{r_{1}}{n}, \frac{r_{2}}{n}, 0\right)$ in the three complex planes paramaterized by $z^{1}, z^{2}, z^{3}$ transverse to the D3-branes (with $r_{1} \pm r_{2}$ even so that the orbifold acts as a $Z_{n}$ on all spinors). The quiver gauge theory has a gauge group $S U(N)^{n} \equiv \prod_{k=1}^{n} S U(N)_{k}$ with one complex scalar corresponding to $Z^{3}$ transforming in the adjoint $\Sigma_{k}\left(\mathbf{N}^{2}-\mathbf{1}\right)_{k}$. The complex scalar corresponding to $Z^{1}$ transforms in the bifundamental representation $\sum_{k=1}^{n}\left(\mathbf{N}_{\mathbf{k}}, \overline{\mathbf{N}}_{\mathbf{k}+\mathbf{r}_{1}}\right)$, and that corresponding to $Z^{2}$ transforms in the bifundamental representation $\sum_{k=1}^{n}\left(\mathbf{N}_{\mathbf{k}}, \overline{\mathbf{N}}_{\mathbf{k}+\mathbf{r}_{\mathbf{2}}}\right)$. Half of the fermions transform in the bifundamental representation $\sum_{k=1}^{n}\left(\mathbf{N}_{\mathbf{k}}, \overline{\mathbf{N}}_{\mathbf{k}+\frac{\mathbf{r}_{1}+\mathbf{r}_{\mathbf{2}}}{2}}\right)$, and the other half transform in the bifundamental representation $\Sigma_{k=1}^{n}\left(\mathbf{N}_{\mathbf{k}}, \overline{\mathbf{N}}_{\mathbf{k}+\frac{\mathbf{r}_{1}-\mathbf{r}_{2}}{2}}\right)$. From this one obtains the effective potential

$$
\begin{aligned}
& V_{e f f}=\frac{g_{Y M}^{4}}{8 \pi^{2}} \sum_{a, b=1}^{N}\left[\frac{1}{2} \sum_{k=1}^{n}\left|x_{k}^{a}-x_{k}^{b}\right|^{4} \log \frac{\left|x_{k}^{a}-x_{k}^{b}\right|^{2}}{\tilde{M}^{2}}+\frac{1}{4} \sum_{k=1}^{n}\left|x_{k}^{a}-x_{k+r_{1}}^{b}\right|^{4} \log \frac{\left|x_{k}^{a}-x_{k+r_{1}}^{b}\right|^{2}}{\tilde{M}^{2}}+\right. \\
& +\frac{1}{4} \sum_{k=1}^{n}\left|x_{k}^{a}-x_{k+r_{2}}^{b}\right|^{4} \log \frac{\left|x_{k}^{a}-x_{k+r_{2}}^{b}\right|^{2}}{\tilde{M}^{2}} \\
& \left.-\frac{1}{2} \sum_{k=1}^{n}\left|x_{k}^{a}-x_{k+\frac{r_{1}+r_{2}}{2}}^{b}\right|^{4} \log \frac{\left|x_{k}^{a}-x_{k+\frac{r_{1}+r_{2}}{2}}^{b}\right|^{2}}{\tilde{M}^{2}}-\frac{1}{2} \sum_{k=1}^{n}\left|x_{k}^{a}-x_{k+\frac{r_{1}-r_{2}}{2}}^{b}\right|^{4} \log \frac{\left|x_{k}^{a}-x_{k+\frac{r_{1}-r_{2}}{2}}^{b}\right|^{2}}{\tilde{M}^{2}}\right]
\end{aligned}
$$

Finally we note that for orbifolds which act freely on the $S^{5}$, with nontrivial rotation angles $2 \pi\left(r_{1} / n, r_{2} / n, r_{3} / n\right)$ on the coordinates $z^{1}, z^{2}, z^{3}$, there are no adjoint scalars and no Coulomb branch. This follows geometrically from the fact that the D-branes span the same dimensions as the orbifold plane and cannot move apart into separate fractional branes at the fixed point. We will return to this in $\S 3$.

\section{Tachyon Condensation and QCD}

We have seen in the above section that the quiver theories corresponding to orbifolds with fixed points on the $S^{5}$ develop a Coleman-Weinberg potential on the classical moduli space at one loop, and we will see in this section that there are interesting unstable directions in which twisted operators get VEVs. 


\subsection{Counting Powers of $N$}

Let us first clarify and interpret in terms of the gravity side the $N$-dependence of the results (2.2) (2.15) 2.16).8 Let us first determine the N-dependence of the 1-loop potential term in the field theory. In all of the orbifold theory potentials we have derived, as noted for the Type 0 case in [9], the coefficient of the logarithmically divergent 4-point interaction among the scalars contains no powers of $N$ beyond that in the factor of $g_{Y M}^{4}$ after terms of the form $g_{Y M}^{4} N \Sigma_{a}\left|x_{a}\right|^{4}$ cancel out of $V_{e f f}$, leaving terms proportional to $g_{Y M}^{4}\left(\operatorname{Tr} X_{1}^{i} X_{1}^{j}-\operatorname{Tr} X_{2}^{i} X_{2}^{j}\right)^{2}$ and $g_{Y M}^{4}\left(\operatorname{Tr} X_{1}^{2}-\operatorname{Tr} X_{2}^{2}\right)^{2}$ at the level of four-point graphs. Let us rescale the $X^{\prime}$ 's so that a factor of $1 / g_{Y M}^{2}=N / \lambda^{\prime} t_{H o o f t}$ appears multiplying the whole tree-level action. Then $g_{Y M}^{2}$ counts loops, and the one-loop potential scales like $g_{Y M}^{0}=1$, down by a factor of $g_{Y M}^{2} \sim 1 / N$ from tree level.

With this normalization of the fields, correlation functions of the single-trace operator $N \operatorname{tr} X^{2}$ scale like $N^{2}$ plus terms subleading in $1 / N^{2}$. These correspond to connected genus-zero amplitudes involving single-particle states on the gravity side [四] [5].

Normalizing operators of the form $\left(\operatorname{tr} X^{2}\right)^{2}$ with a power of $N^{2}$ :

$$
\mathcal{O}_{\text {double-trace }}=N^{2}\left(\operatorname{tr} X^{2}\right)^{2}
$$

we obtain $l$-point correlation functions of the $\mathcal{O}_{\text {double-trace }}$ which scale like $N^{2 l}$ in the free theory, corresponding to $l$ disconnected genus zero diagrams describing $l$ strings propagating across the AdS. This is in line with the interpretation of multitrace operators as multiparticle states on the gravity side in the unperturbed theory.

As discussed below (2.1), the twisted operators in our theory are of the form $\operatorname{tr} X_{k}^{2}-$ $\operatorname{tr} X_{k^{\prime}}^{2}$. Because they transform non-trivially under the quantum symmetry of the orbifold, terms in the Lagrangian linear in these operators are not generated dynamically, but terms quadratic in these operators which are invariant under the quantum symmetry are (they are implicit in the potentials (2.2) (2.15) calculated out along the Coulomb branches in the last section). These are double-trace operators, which are thought to correspond to multiparticle excitations of the dual gravity theory [4][5]. As just discussed, as they appear at one-loop these contributions scale like

$$
\delta S \sim \int\left(\operatorname{tr} X^{2}\right)^{2}
$$

8 The results on $N$-dependence here, some aspects of which appear in [9], were developed in discussions with O. Aharony. 
Now consider adding a contribution of the order $\left(\operatorname{tr} X^{2}\right)^{2}$ to the action (as occurs dynamically in our theory (3.2)). Bringing down a power of (3.2) into correlation functions, one finds the leading- $\mathrm{N}$ effect from factorized terms of the form

$$
\left\langle:\left(\operatorname{Tr} X_{k}^{2}-\operatorname{Tr} X_{k^{\prime}}^{2}\right)^{2}: \mathcal{O}_{1}, \ldots, \mathcal{O}_{l}\right\rangle \sim\left\langle\left(\operatorname{Tr} X_{k}^{2}-\operatorname{Tr} X_{k^{\prime}}^{2}\right) \mathcal{O}_{1}, \ldots, \mathcal{O}_{l^{\prime}}\right\rangle\left\langle\left(\operatorname{Tr} X_{k}^{2}-\operatorname{Tr} X_{k^{\prime}}^{2}\right) \mathcal{O}_{l^{\prime}+1}, \ldots, \mathcal{O}_{l}\right\rangle
$$

where we have replaced $\left(\operatorname{Tr} X^{2}\right)^{2}$ in $(3.2)$ with the more precise form $\left(\operatorname{Tr} X_{k}^{2}-\operatorname{Tr} X_{k^{\prime}}^{2}\right)^{2}$ we have for deformations of our theories. These go like $N^{2}$. However if all the $\mathcal{O}_{1}, \ldots, \mathcal{O}_{k}$ are untwisted operators, then each factor in the factorized leading- $\mathrm{N}$ contribution vanishes, and one is left with an effect that is down by $1 / N^{2}$ from genus-zero effects. This is in accord with large- $\mathrm{N}$ inheritance on the gravity side [1] and the field theory side [31], which ensures that at large $\mathrm{N}$ the correlators of untwisted operators are the same as in the $\mathcal{N}=4$ theory. The twisted operators do not exist in the parent theory, and are not constrained by inheritance.

It is interesting that a mass scale $\tilde{M}$ appears in correlators of twisted operators at genus zero. In particular, the couplings of the double trace operators $\Sigma_{k}\left(\operatorname{Tr} X_{k}^{2}-\operatorname{Tr} X_{k^{\prime}}^{2}\right)^{2}$ have nontrivial beta functions at one-loop (as can be seen from the four-point function contribution to the effective potentials calculated in $\S 2$ ). So even before we go out on the Coulomb branch, the theory is nonconformal at leading order in $\mathrm{N}$ in a nontrivial regime of $\lambda_{t \text { thooft }}$. This is invisible to the untwisted operators alone at this order in $\mathrm{N}$, in accord with [31]. Even so, this is puzzling because of the general arguments advanced in [1] for the large- $\mathrm{N}$ conformality of these theories. In the next subsection, we will discuss this puzzle and several possible resolutions which it will be interesting to pursue once we have pushed the relevant technology to the necessary level.

\subsection{Orbifolding and Symmetries: A Puzzle}

As discussed in [1], there is a fairly general reason to believe that orbifold field theories should have conformal invariance at large $\mathrm{N}$, including the physics of twisted operators. 8 The worldsheet sigma model describing strings propagating on the parent space $A d S_{5} \times S^{5}$ has a symmetry corresponding to the $S O(4,2)$ isometries of the $A d S_{5}$, which commutes with the $S O(6)$ of the $S^{5}$ and in particular commutes with an action of $\Gamma \subset S O(6)$ on $S^{5}$. This symmetry commutes with the Hamiltonian of the worldsheet

9 We thank T. Banks and S. Kachru for discussions of this. 
theory, and therefore all of its correlation functions respect it. This parent sigma model has many operators, some subset of which $\left\{V_{\text {parent }}\right\}$ constitute mutually local dimension $(1,1)$ vertex operators describing physical string states. When we orbifold, for example by the $Z_{n}$ actions we are considering in this paper, we include only those vertex operators $\left\{V_{\text {untwisted }}\right\} \equiv\left(\left\{V_{\text {invariant }}\right\} \subset\left\{V_{\text {parent }}\right\}\right)$ which are invariant under the orbifold group action. Having done this one can (and should at the one-loop level) add "twisted" operators which are further operators from the set of operators in the parent sigma model which are mutually local with respect to the reduced set of operators $\left\{V_{\text {untwisted }}\right\}$. So finally $\left\{V_{\text {orbifold }}\right\}=\left\{V_{\text {untwisted }}+V_{\text {twisted }}\right\}$ gives the full set of vertex operators for the orbifold theory. The Hamiltonian of the full worldsheet sigma model is the same in all of these theories, and commutes with the $S O(4,2)$. So the orbifold theory should have this symmetry and the QFT dual to it by AdS/CFT should be conformally invariant for all $\lambda_{\prime} t_{\text {Hooft }}$ at leading order in the $1 / N^{2}$ expansion. This argument appears rather general (though unforeseen subtleties involving RR fields may render it inapplicable to our case).

On the other hand, at weak coupling in the quiver gauge theory one finds (as we have discussed) nontrivial beta functions for the double-trace quartic scalar interactions of the form $\Sigma_{k, k^{\prime}} \lambda\left(\operatorname{Tr} X_{k}^{2}-\operatorname{Tr} X_{k^{\prime}}^{2}\right)^{2}$. Although it is made out of twisted operators, this contribution to the Lagrangian does not itself transform under the quantum symmetry and if we do not condense the twisted operators we should not have left the orbifold point.

We do not yet know the resolution of this puzzle, but can see several interesting possibilities (which are not all mutually exclusive):

(1) The above argument about the symmetries is correct and applies to the RR sigma models of interest here. This would suggest that there is a line of fixed points corresponding to the radius of $A d S_{5} \times S^{5} / Z_{n}$. Since starting at weak coupling on the field theory side there is not such a fixed line, this line of fixed points would have to be fundamentally strongly coupled.

Then the theories we consider here, with running $\lambda_{a b}^{i j}$, are deformations away from the line of CFTs dual to the standard orbifold of $A d S_{5} \times S^{5}$. But these theories share many properties with the standard orbifold, in particular the quantum symmetry and the inheritance of untwisted operator correlation functions at large N. Therefore even if (1) is true we feel it is important to understand the gravity-side description of our (perhaps nonstandard) orbifold models. This leads to possibility 
(2) The double-trace operators in the effective Lagrangian of our models correspond to a novel type of worldsheet string theory on the gravity side, such as the one under investigation independently in a supersymmetric context with marginal double-trace perturbations [35].10 This novel string theory, if it exists and applies to our models here, may not have all the properties required for the above symmetry argument. As discussed above, a relative of this possibility is the possibility that RR sigma models do not satisfy the assumptions in the symmetry argument presented above.

Finally, there is always the possibility

(3) Phase transitions and/or other unconstrained non-supersymmetric dynamics ruin the application of the duality to this non-supersymmetric context. Because we began with a parent system with two dual descriptions, the procedure applied to one of them producing the orbifold theory ought to have a translation into the dual variables if it exists nonperturbatively. This translation to the dual may not be a standard orbifold construction, however, which may relate to point (2). Indeed a phase transition in this type of system is suggested by the large-radius duality map, which maps the tachyon to a complex-dimension operator in the field theory [1]. It would be very interesting to understand better what this means for the duality, but in this paper we will continue to focus on the small-radius (weak 't Hooft coupling) regime.

Because of the RR fields and strong coupling issues, establishing the precise resolution of this puzzle appears out of reach of current technology, and we will leave it for future work. We think it is likely that there is a resolution (perhaps along the lines of (1) and/or (2)) which preserves the duality and teaches us something new about the gravity side, and we will proceed with our analysis on the assumption that the duality holds. In particular, our analysis has generated further concrete evidence in favor of the duality (in addition to generating the puzzle discussed in this subsection). However, possibility (3) should be kept in mind.

\subsection{Tachyons and AdS/CFT Duality}

It has been suggested [3] [1] that the Type 0 tachyon is lifted at small $A d S$ radius to satisfy the Breitenlohner-Freedman bound [36. Heuristically this might be expected from the fact that the $A d S$ curvature reaches string scale for small enough 't Hooft coupling, so that a string-scale tachyon need not violate the bound [3]. This sort of behavior has

10 We thank O. Aharony and M. Berkooz for sharing with us their ideas on this. 
been seen in the $A d S_{3}$ context in [37. Further, the twisted operator $\operatorname{tr} F_{1}^{2}+D X_{1} D X_{1}-$ $F_{2}^{2}-D X_{2} D X_{2}$, to which the tachyon couples directly at large radius, is actually slightly irrelevant at weak coupling [11], which according to an uncorrected extrapolation of the large radius duality map would translate to a non-tachyonic mass in the bulk gravity theory.

However, we have seen that the weakly coupled dual field theory has instabilities in the potential at leading order in $1 / N^{2}$ which cause certain twisted operators (which have the same discrete quantum numbers as the large-radius tachyons) to condense, either directly or via tunneling depending on the small- $X$ behavior of the potential. That the instability appears at genus zero shows that it persists even in the strict large-N limit. This demonstrates an instability of the string theory which causes modes from the orbifold twisted sectors to condense even at small radius.

As we have discussed, because of the running couplings in the theory, our one-loop analysis is not sufficient to determine whether the instability is perturbative or requires a non-perturbative tunneling process to access. If it is non-perturbative, the situation is reminiscent of those described in [15], where a tachyonic instability in one limit of moduli space appears to turn into a non-perturbative instability mediated by a gravitational instanton in another limit.

\subsection{Patterns of Symmetry Breaking}

In the remainder of this section we will provide a preliminary discussion of the physics that results when the twisted operator VEVs turn on. We will begin with some heuristic intuition from the gravity side, and then analyze concretely some aspects of the Higgs structure of the model given the scalar potentials calculated in the previous section.

On the gravity side, we expect perturbative tachyon condensation to produce a subcritical dimension target spacetime [12] 113]. This is because the zero-momentum tachyon vertex operator is a relevant operator on the string worldsheet. The worldsheet beta function equations are then satisfied by a nontrivial field configuration for the dilaton, metric, and other string fields; in particular dilaton gradients contribute effective central charge to compensate for that lost by going to a subcritical dimension (as occurs for example in the case of a linear dilaton with flat string-frame metric) [12]. In the context of the AdS/CFT correspondence, the dimensions of the $S^{5} / Z_{n}$ and the radial direction of AdS arose from the directions transverse to the D3-branes, which are parameterized by worldvolume scalars. It is natural to expect therefore that losing and/or deforming the $S^{5}$ and 
radial dimensions would correspond to losing the scalars in the dual quiver gauge theory, and perhaps also the fermions which also transform under $S O(6)$ rotations.

In situations with non-perturbative instabilities on the gravity side [15] one also has a sense in which degrees of freedom are lost, as one "tunnels to nothing".11 In our situation, as we will discuss shortly, one does not always expect to decay to nothing, but one can decay to something which is in some sense less than what one had to begin with: from the full quiver gauge theory to a long-distance sector with pure glue QCD.

Let us discuss some patterns of symmetry breaking that emerge from our potentials (2.2)(2.15)(2.16). There are instabilities in the effective potential correponding to VEVs for twisted operators in the gauge theory, manifested in the D-brane language convenient for the calculations in $\S 2$ by relative motion of fractional branes along the orbifold plane described by VEVs for diagonal entries of the adjoint scalar matrices. Let us investigate the effect of turning on these VEVs.

Let us analyze the Type 0 case (2.2) for simplicity; similar patterns will emerge in the higher $Z_{n}$ cases and can be analyzed in a similar way. Consider the direction in field space in which $X_{1}^{i}$ gets a VEV

$$
\left\langle X_{1}^{i}\right\rangle=\operatorname{diag}\left(\rho_{1}^{i}, \rho_{2}^{i}, \ldots, \rho_{N-1}^{i},-\rho_{1}^{i}-\rho_{2}^{i}-\ldots-\rho_{N-1}^{i}\right)
$$

which satisfies the $S U(N)$ condition

$$
\Sigma_{a=1}^{N} \rho_{a}=0
$$

and in which $\left\langle X_{2}^{i}\right\rangle=0$.

Plugging this into the effective potential (2.2), we obtain

$$
V\left(\overrightarrow{\rho_{a}}\right) \sim g_{Y M}^{4}\left(\Sigma_{a, b}\left|\vec{\rho}_{a}-\vec{\rho}_{b}\right|^{4} \log \frac{\left|\vec{\rho}_{a}-\vec{\rho}_{b}\right|^{2}}{\tilde{M}^{2}}-2 N \Sigma_{a}\left|\vec{\rho}_{a}\right|^{4} \log \frac{\left|\vec{\rho}_{a}\right|^{2}}{\tilde{M}^{2}}\right)
$$

where we have replaced the transverse $R^{6}$ index $i$ by vector notation.

Let us consider the minimization of this potential with respect to the $\rho_{a}^{i}$. The first term in (3.6) describes the force between "electric" branes (those with $S U(N)_{1}$ on their worldvolume). The second term describes the force between the electric branes and the

11 From other points of view one appears to tunnel to flat space via a Schwinger effect [28] [29], a situation whose interpretation and whose relation to our results here would be very interesting to clarify. 
"magnetic" branes (those with $S U(N)_{2}$ on their worldvolume) which are all sitting at the origin, $\vec{X}_{2}=0$. At sufficiently small distances between the branes, the former is repulsive and the latter is attractive.

There is a relatively simple configuration where the $\vec{\rho}_{a}$ are arranged symmetrically (equally spaced) on an $S^{5}$ of radius $\rho$. This satisfies the $S U(N)$ constraint (3.5). It is an extremum of the effective action in the angular directions. By playing the mutual repulsion of the electric branes against their attraction to the magnetic branes at the center, we will find a minimum for the radial mode $\rho$, generalizing the one along the direction $\left\langle X_{1}^{i}\right\rangle=\operatorname{diag}(\rho, 0, \ldots, 0,-\rho)$ discussed in [9].

Approximating the sum over branes indexed by $a$ by an integral over the angles of the $S^{5}$, we obtain an approximate form of the potential which will be sufficient to indicate the presence of the anticipated minimum:

$$
\frac{V}{g_{Y M}^{4}} \sim \int d \Omega_{1} d \Omega_{2}\left|\vec{\rho}\left(\Omega_{1}\right)-\vec{\rho}\left(\Omega_{2}\right)\right|^{4} \log \frac{\left|\vec{\rho}\left(\Omega_{1}\right)-\vec{\rho}\left(\Omega_{2}\right)\right|^{2}}{\tilde{M}^{2}}-2 N \int d \Omega_{1}\left|\vec{\rho}\left(\Omega_{1}\right)\right|^{4} \log \frac{\left|\vec{\rho}\left(\Omega_{1}\right)\right|^{2}}{\tilde{M}^{2}}
$$

Using this, separating the radius $\rho$ of the sphere of fractional D-branes from the angular variables, we obtain

$$
V(\rho) \sim N^{2} g_{Y M}^{4} \rho^{4} \log \left(\frac{\rho^{2} e^{379 / 240}}{\tilde{M}^{2}}\right)
$$

The potential (3.8) has a minimum at $\rho$ of order $\tilde{M}$,

$$
\rho_{\text {min }}^{2}=\tilde{M}^{2} e^{-1 / 2} e^{-379 / 240}
$$

as in our earlier discussion of the Coleman-Weinberg potential. We have therefore balanced the attractive and repulsive forces as anticipated. In particular, one finds the force between the electric branes and the magnetic branes is attractive in this regime. Because the forces grow with distance, this suggests that the magnetic branes at the origin are stable against small fluctuations, which means the $X_{2}^{i}$ scalars are massive. Indeed this follows from an analysis of small fluctuations in the $\vec{x}_{2 b}$ directions, as follows.

Expanding (2.2) around the background symmetric distribution of $\vec{\rho}_{a}$, we find the following mass terms for the $x_{2, b}^{i}$ :

$$
-2 g_{Y M}^{4} \Sigma_{a, b}\left(2\left|\vec{x}_{2, b}\right|^{2}\left|\vec{\rho}_{a}\right|^{2} \log \frac{\left|\vec{\rho}_{a}\right|^{2} e^{1 / 2}}{\tilde{M}^{2}}+4 x_{2, b}^{l} x_{2, b}^{m} \rho_{a}^{l} \rho_{a}^{m} \log \frac{\left|\vec{\rho}_{a}\right|^{2} e^{3 / 2}}{\tilde{M}^{2}}\right)
$$


Summing over the spherically distributed $\vec{\rho}_{a}$, the off-diagonal terms in the mass matrix sum to zero and we see that the diagonal terms are nonzero and positive at the minimum (3.9) (since $\log \frac{\rho_{\min }^{2} e^{1 / 2}}{M^{2}}=-379 / 240<0$ and $\log \frac{\rho_{\min }^{2} e^{3 / 2}}{M^{2}}=1-379 / 240<0$ ). 12

We have now accumulated enough information to determine the effect on the gauge theory of turning on this VEV. It breaks one $S U(N)$ to $U(1)^{N-1}$ and leaves the other $S U(N)$ intact. From (2.1) one finds that all the fermions get masses once our VEVs (3.4) are turned on. As we have just seen, the scalars $X_{2}^{i}$ get positive mass squared.

The angular fluctuations of the $X_{1}^{i}$ on the other hand are dominated by repulsive interactions between the electric branes, so these fluctuations appear to be unstable. Once this configuration of twisted VEVs in the gauge theory is turned on, the long-distance physics of the gauge theory consists of QCD plus decoupled U(1) factors and unstable scalars.

There is another configuration in which QCD sectors emerge at low energies without instabilities in the other sectors; this is a likely endpoint of the spherical configuration we began with. It is another natural generalization to large $N$ of the instability discussed in [9]. Consider again $\left\langle\vec{X}_{2}=0\right\rangle$. Take $\left\langle X_{1}\right\rangle=\operatorname{diag}(\vec{r}, \vec{r}, \ldots, \vec{r},-\vec{r}, \ldots,-\vec{r})$ where the first $N / 2$ diagonal entries are $\vec{r}$ and the last $N / 2$ entries are $-\vec{r}$. Geometrically, this describes $N / 2$ electric branes at $-\vec{r}$ and $N / 2$ electric branes at $+\vec{r}$, with $N$ magnetic branes at the origin.

In this case, there is a minimum at

$$
\frac{r_{\min }^{2} e^{1 / 2}}{\tilde{M}^{2}}=2^{-8 / 3}
$$

about which the fluctuations of both $\vec{X}_{1}$ and $\vec{X}_{2}$ work out to be massive, which again is consistent with naive expectation from the signs of the forces in the vicinity of the minimum (3.11). The Yukawa couplings (2.1) yield masses for all the bifundamental fermions in this configuration.

This more stable configuration leaves, on the gauge theory side at distances long compared to the masses of the scalars and fermions, a pure glue QCD sector with gauge group $S U(N)$, decoupled from two others with gauge groups $S U(N / 2)$ and a relative $U(1)$ factor.

12 Note that there is no coupling-constant dependence in the computation of the signs of forces and scalar $\mathrm{m}^{2}$ 's which depend only on the values of order one numbers arising from the geometry of the configuration at the minimum (3.9). 
Because of the limited range of validity of the 1-loop calculation of $V_{e f f}$, we do not know if the potential is bounded or unbounded from below at large $\langle X\rangle$. If it turns out to be unbounded, it is tempting to suggest that for infinitely large VEVs for the twisted operators in the gauge theory, the theory may reduce to separate pure glue QCD plus decoupled $U(1)$ sectors. However because of the long-range forces on this fractional Dbrane branch of the gauge theory, it is not clear what the masses of the $X_{2}$ 's will be as a function of the VEVs of the $X_{1}$ 's, and it is logically possible that the $X_{2}$ scalars would come back down to zero mass and/or become unstable as $X_{1}$ increases beyond the regime of validity of our current calculation.

In our $Z_{n}$ orbifolds, before going out on the Coulomb branch the gauge group is $S U(N)^{n} \equiv \Pi_{k=1}^{n} S U(N)_{k}$. Turning on VEVs for the diagonal elements of the $X$ 's similarly leads to the near-horizon limit of various fractional D-brane configurations whose lowenergy theories involve gauge symmetry breaking and some massive and/or decoupled scalar and fermion matter. It would be interesting to classify all the possible behaviors in arbitrary $Z_{n}$ orbifold models based on the potentials derived in the last section, but we will leave that for future work.

It is not clear from this analysis whether this M-theory dual to the remaining gauge theory will be a perturbative string theory. 13 Indeed in ordinary large-radius string backgrounds the tachyon mixes with the dilaton, and its condensation leads to strong dilaton gradients; the analogous phenomenon should be expected in our small-radius AdS/CFT system. (Correspondingly on the gauge theory side, the VEVs for twisted operators that we have turned on can induce large renormalizations of all the couplings in the gauge theory.) Even before turning on VEVs for twisted operators along the unstable directions, a novel kind of string theory may be required on the gravity side of a dual pair in which the field theory is deformed by a double-trace operator of this kind, as discussed in $§ 3.2$ [35].

In any case, we have arrived at an interesting answer via AdS/CFT duality to the question of what can happen when one condenses a tachyon in closed string theory: in this system, it rolls to the gravity dual of a gauge theory with less symmetry and reduced matter content, but sometimes retaining pure glue QCD factors in the infrared.

As discussed above, condensing the tachyon is expected to lead to a subcritical matter sector on the string worldsheet, and we have just learned that the corresponding process

13 The results [38 perhaps suggest otherwise. 
on the gauge theory side can lift or decouple the extra matter and gauge fields beyond pure glue QCD. Noncritical string theory was conjectured to be dual to QCD in [16]. Our results provide some further evidence in this direction.

\section{Freely Acting Orbifolds and Tachyon-freedom}

Consider an orbifold group $\Gamma \subset S O(6)$ which fixes an isolated point in $R^{6}$. In the presence of $N$ D3-branes centered at the fixed point, the spacetime geometry blows up into a near-horizon region which is completely smooth, with the orbifold acting freely on the $S^{5}$.

Because the orbifold fixes an isolated point in $R^{6}$, the codimension of the singularity is the same as the codimension of the D3-branes, so the spacetime has no directions along which fractional branes could separate. Correspondingly, the scalars in the resulting quiver gauge theory are all in bifundamental representations, in contrast to the above non-freelyacting cases where the scalars describing motion along the orbifold fixed locus remained in the adjoint. Thus, for freely acting orbifolds, there is no Coulomb branch.

Since in these cases the classical moduli space does not include a branch where twisted states can develop a VEV, the theory will remain stable to all orders in $\lambda_{t H o o f t}$. We therefore suspect that there are no twisted instabilities for any radius (any 't Hooft coupling $\left.\lambda^{\prime} t_{\text {Hooft }}\right)$ in this system, though there is a logical possibility that one develops at a $\lambda^{\prime} t_{H o o f t}$ of order one. (If so, it would have to disappear again for large $\lambda_{\prime} t H o o f t$ as discussed above. This result is a new prediction for the gravity description at small radius. 14

\section{5. $Z_{n} \times Z_{n}$ Orbifolds with Discrete Torsion}

In more general orbifolds than those we have considered so far, such as $Z_{n} \times Z_{n}$ orbifolds, one can project out the lowest-lying twisted-sector tachyons with a nontrivial choice of discrete torsion. However, at large radius, tachyonic modes dressed with angular

14 Very naive gravity-side intuition might have suggested that a tachyon would arise at small radius, since the positive mass squared of twisted states at large radius is driven by their winding energy around the $S^{5} / \Gamma$, and when the $S^{5} / \Gamma$ becomes small the winding energy would appear to be negligible. However, the substringy dynamics of curved Ramond backgrounds is hardly a place where naive intuition applies. Our QFT analysis of the lack of instability in this system at small radius is a prediction for the worldsheet $R R$ sigma model. 
momentum along the $S^{5} / \Gamma$ will survive this projection. It is interesting to consider whether this instability persists at small radius (weak 't Hooft coupling) in these theories.

In this section we will study in particular a $\left(C / Z_{3}\right) \times\left(C / Z_{3}\right)$ orbifold with nontrivial discrete torsion. Let the first $Z_{3}$, generated by $g_{1}$, act on the $z^{1}$ direction, and the second generated by $g_{2}$ on the $z^{2}$ direction, with the third complex plane parameterized by $z^{3}$ left invariant. The $g_{1}$ twisted sector vacuum energy is $-1 / 3$, as is that of the $g_{2}$ twisted sector, and in the absence of nontrivial discrete torsion these states correspond to physical tachyons in spacetime. A nontrivial choice of discrete torsion projects out each of these vacua ( $g_{1}$ projecting out the vacuum in the $g_{2}$ twisted sector and vice versa). However, there are momentum states invariant under the both $g_{1}$ and $g_{2}$ which still have tachyonic masses $m^{2}<0$ in spacetime.

Naive intuition might suggest that these momentum-mode tachyons may get lifted as we go toward small radius since the momentum contribution to the $m^{2}$ of the state grows as the radius shrinks. Again naive intuition is liable to fail in these highly curved Ramond backgrounds, and as in the previous examples, the QFT instability analysis is the appropriate method for answering this question at small radius given the limitations of current technology on the gravity side.

The worldvolume theory of D-branes on orbifolds with discrete torsion was worked out in [39] (and studied in the context of AdS/CFT in [40]). The result for our case in particular is as follows. The theory is an $S U(3 N)$ gauge theory with three complex scalars $Z^{1,2,3}$ and four Weyl spinors $\chi^{1, \ldots, 4}$ in the adjoint, i.e. a theory with the field content of $\mathcal{N}=4 S U(3 N)$ SYM. The interactions, however, differ from those of the $\mathcal{N}=4$ theory. The quartic scalar couplings involving $Z^{1}$ and $Z^{2}$ are deformed from the usual commutators to take the form:

$$
\begin{aligned}
& \mathcal{L}_{\text {scalar }}=\operatorname{tr}\left[\left(Z^{1} Z^{2}-\alpha Z^{2} Z^{1}\right)\left(Z_{\overline{2}} Z_{\overline{1}}-\alpha^{-1} Z_{\overline{1}} Z_{\overline{2}}\right)\right] \\
& \operatorname{tr}\left(\left[Z^{1}, Z^{3}\right]\left[Z^{1}, Z^{3}\right]^{\dagger}\right)+\operatorname{tr}\left(\left[Z^{2}, Z^{3}\right]\left[Z^{2}, Z^{3}\right]^{\dagger}\right)
\end{aligned}
$$

where $\alpha=e^{2 \pi i / 3}$. Similarly the Yukawa couplings are deformed to the form

$$
\begin{aligned}
& \alpha^{-1} \Gamma_{1}^{\alpha \beta} \operatorname{tr}\left[\chi^{\alpha}\left(Z^{1} \chi^{\beta}-\alpha^{-1} \chi^{\beta} Z^{1}\right)\right]+\alpha \Gamma_{2}^{\alpha \beta} \operatorname{tr}\left[\chi^{\alpha}\left(Z^{2} \chi^{\beta}-\alpha \chi^{\beta} Z^{2}\right)\right] \\
& +\Gamma_{3}^{\alpha \beta} \operatorname{tr}\left(\chi^{\alpha}\left[Z^{3}, \chi^{\beta}\right]\right)
\end{aligned}
$$


The Coulomb branch describing fractional branes is parameterized by diagonal $Z^{3}$ matrices. We can now immediately observe a difference between this case and the cases discussed in $§ 2$. Namely, the one-loop Coleman-Weinberg potential will be absent here, since all the tree-level vertices involving $Z^{3}$ are exactly the same as in an $S U(3 N) \mathcal{N}=4$ theory. On the other hand, higher-loop contributions to the effective potential of the gauge theory mix $Z^{3}$ with all the other fields, and we expect such contributions will get generated. It would be interesting to explore their signs to see if an instability exists in this case at higher orders in $\lambda^{\prime}$ tHooft.

\section{Discussion and Future Directions}

In this paper we have identified global instabilities in certain weakly-coupled nonsupersymmetric gauge theories whose AdS/CFT duals contain twisted-sector tachyons at large radius. These instabilites, which induce VEVs for twisted field theory operators, appear at one-loop in the gauge theory and genus zero in the string theory, though their effect on untwisted operators is suppressed by factors of $\left(1 / N^{2}\right)$, as expected from large-N inheritance.

At higher orders in $1 / N^{2}$ there will be a rich set of dynamically generated contributions to the effective action which are not constrained by large- $\mathrm{N}$ inheritance from the parent $\mathcal{N}=4$ theory. This can (and presumably does) include quadratically divergent scalar masses (as well as quartically divergent vacuum energy which does not affect the QFT dynamics). It would be interesting to calculate these effects and understand their description on the gravity side of the correspondence. These finite- $\mathrm{N}$ effects can have a dramatic effect on the matter content and dynamics, and it is necessary to calculate these in order to understand the finite- $\mathrm{N}$ system. Some interesting perturbative calculations in these theories were done for example in [41]. While we feel such an analysis is further motivated by our work here, it is somewhat subtle to carry out since the QFT couplings appropriate to the gravity dual may themselves be shifted from the orbifold values by contributions of order $1 / N^{2} .15$

As discussed in $\S 2$, it would be very interesting to ascertain the behavior of the effective potential near the origin of the Coulomb branch. It would also be interesting to see whether a higher-loop analysis leads to persistant instability at large $\langle X\rangle$, and to study

15 We thank M. Strassler for reminding us of this difficulty. 
the meaning of (and possible constrints on) the quiver theory renormalization conditions from the gravitational dual. These last issues mirror the difficulty on the gravity side of determining the form of the tachyon potential when the tachyon $\mathrm{VEV}$ is large and mixes strongly with other string fields.

One important generalization to consider is a case where quark flavors survive the tachyon condensation process, so that we get more than just the pure glue QCD theory. Recall that in our tachyon condensation process in $\S 3$ the fermions decoupled and/or became massive as the twisted operator's VEV turned on. A case where flavors survive may well involve a second set of D-branes in addition to the D3-branes contributing the $S U(N)$ gauge group, so that the gravity side has open strings as well as closed strings. It would be interesting to perform a general analysis of symmetry breaking patterns for the $Z_{n}$ orbifolds considered here and more general ones; the configurations we discussed in $\S 3.4$ are particularly simple and there may be a rich set of interesting possibilities implicit in the potentials 2.2 (2.15) (2.16).

It would be interesting to explore the tachyon potential in closed string field theory in this system, and compare the Coleman-Weinberg potential to the closed-string analogues of formulas in [27] for the tachyon potential in open string field theory. This is out of range of current technology, and our QFT calculations are simply predictions for the behavior of the appropriate gravity side sigma model.

It would also be interesting to study the tachyon potential on the gravity side at large radius, to see what happens to the $S^{5} / \Gamma$ and to the RR fields upon tachyon condensation in that regime. We may be able to get a handle on this by studying the geometry of fractional D-branes splitting apart, via nonsuper-gravity at large radius. It would also be very interesting to explore potential relations to the work of [30] and [29]. With respect to the latter, one would need to repeat our analysis of D3-branes in the Type 0B theory for the (nonconformal) even-dimensional D-branes of the Type 0A theory, where the conjectured dualities and instabilities of [29] might apply most directly.

In general, it is important to improve our understanding of the gravity side of the duality (and the duality map) in order to resolve the puzzle of the violation of conformal invariance on the field theory side discussed at length in $§ 3.2$.

Finally, it would be interesting to study further examples of tachyonic and nontachyonic non-supersymmetric AdS/CFT duals, to see how general the pattern found here of large radius instabilities persisting to small radius proves. We covered a large class of examples in our analysis here, but there are many more cases that could be considered. 


\section{Acknowledgements}

We would like to thank O. Aharony for very useful comments and questions on this project and for sharing with us his insights with M. Berkooz on double-trace operators in an independent context [35]. We would also like to thank T. Banks, M. Berkooz, O. DeWolfe, D. Gross, A. Hashimoto, G. Horowitz, S. Kachru, I. Klebanov, D. Kutasov, A. Lawrence, H. Ooguri, S. Sethi, S. Shenker, M. Strassler, A. Strominger, J. Troost and K. Zarembo for interesting and helpful discussions on this and/or related topics. We thank the participants of the ITP M-theory workshop for their questions and comments during a discussion of an earlier incarnation of this work. Our research is supported in part by the Department of Energy under contract DE-AC03-76SF00098, by NSF grant PHY-9514797, by A DOE OJI grant, and by an Alfred P. Sloan Foundation Fellowship. We are

grateful for the hospitality of the Institute for Theoretical Physics at UCSB, where we receive support from the NSF under grant number PHY-99-07949. The research of A.A. was also supported in part by an NSF Graduate Fellowship. 


\section{References}

[1] S. Kachru and E. Silverstein, "4d conformal theories and strings on orbifolds," Phys. Rev. Lett. 80, 4855 (1998) hep-th/9802183.

[2] A. E. Lawrence, N. Nekrasov and C. Vafa, "On conformal field theories in four dimensions," Nucl. Phys. B 533, 199 (1998) hep-th/9803015; M. Berkooz, "A supergravity dual of a $(1,0)$ field theory in six dimensions," Phys. Lett. B 437, 315 (1998) hepth/9802195.

[3] I. R. Klebanov and A. A. Tseytlin, "A non-supersymmetric large N CFT from type 0 string theory," JHEP9903, 015 (1999) hep-th/9901101; I. R. Klebanov and A. A. Tseytlin, "D-branes and dual gauge theories in type 0 strings," Nucl. Phys. B 546, 155 (1999) hep-th/9811035; I. R. Klebanov and A. A. Tseytlin, "Asymptotic freedom and infrared behavior in the type 0 string approach to gauge theory," Nucl. Phys. B 547, 143 (1999) hep-th/9812089; I. R. Klebanov, N. A. Nekrasov and S. L. Shatashvili, "An orbifold of type 0B strings and non-supersymmetric gauge theories," Nucl. Phys. B 591, 26 (2000) hep-th/9909109.

[4] J. Maldacena, "The Large N Limit of Superconformal Field Theories and Supergravity," ATMP 2 (1998) 231, hep-th/9711200.

[5] S. Gubser, I. Klebanov and A. Polyakov, "Gauge Theory Correlators from Noncritical String Theory," Phys. Lett. B428 (1998) 105, hep-th/9802109; E. Witten, "Anti-de Sitter Space and Holography," ATMP 2 (1998) 253, hep-th/9802150.

[6] S. Kachru, J. Kumar and E. Silverstein, "Vacuum energy cancellation in a nonsupersymmetric string," Phys. Rev. D 59, 106004 (1999) hep-th/9807076.

[7] C. Angelantonj and A. Armoni, "RG flow, Wilson Loops, and the Dilaton Tadpole", Phys. Lett. B 482329 (2000); "Non-Tachyonic type 0B Orientifolds, Nonsupersymmetric Gauge Theories, and Cosmological RG Flow", Nucl. Phys. B 578239 (2000).

[8] M. R. Douglas and G. Moore, "D-branes, Quivers, and ALE Instantons," hepth/9603167.

[9] A. A. Tseytlin and K. Zarembo, "Effective potential in non-supersymmetric SU(N) x SU(N) gauge theory and interactions of type 0 D3-branes," Phys. Lett. B 457, 77 (1999) hep-th/9902095.

[10] S. Coleman and E. Weinberg, "Radiative Corrections As The Origin Of Spontaneous Symmetry Breaking," Phys. Rev. D 7 (1973) 1888.

[11] I. R. Klebanov, "Tachyon stabilization in the AdS/CFT correspondence," Phys. Lett. B 466, 166 (1999) hep-th/9906220.

[12] S. P. de Alwis, J. Polchinski and R. Schimmrigk, "Heterotic Strings With Tree Level Cosmological Constant," Phys. Lett. B 218, 449 (1989). 
[13] I. Antoniadis and C. Kounnas, "Superstring Phase Transition at High Temperature", Phys. Lett. B261 (1991) 369.

[14] S. Kachru, J. Kumar and E. Silverstein, "Orientifolds, RG flows, and closed string tachyons," Class. Quant. Grav. 17, 1139 (2000) hep-th/9907038.

[15] E. Witten, "Instability Of The Kaluza-Klein Vacuum," Nucl. Phys. B 195, 481 (1982); M. Fabinger and P. Horava, "Casimir effect between world-branes in heterotic Mtheory," Nucl. Phys. B 580, 243 (2000) hep-th/0002073,

[16] A. M. Polyakov, "The wall of the cave," Int. J. Mod. Phys. A 14, 645 (1999) hepth/9809057; A. M. Polyakov, "String theory and quark confinement," Nucl. Phys. Proc. Suppl. 68, 1 (1998) hep-th/9711002.

[17] J. A. Minahan, "Asymptotic freedom and confinement from type 0 string theory," JHEP9904, 007 (1999) hep-th/9902074.

[18] J. Polchinski and M. J. Strassler, "The string dual of a confining four-dimensional gauge theory," hep-th/0003136.

[19] E. Silverstein and Y. S. Song, "On the critical behavior of D1-brane theories," JHEP0003, 029 (2000) hep-th/9912244.

[20] V. Niarchos, "Density of states and tachyons in open and closed string theory," hepth/0010154.

[21] F. Bigazzi, "RG flows toward IR isolated fixed points: Some type 0 samples", hepth/0101232.

[22] J. D. Blum and K. R. Dienes, "Strong/weak coupling duality relations for nonsupersymmetric string theories," Nucl. Phys. B 516, 83 (1998) hep-th/9707160.

[23] O. Bergman and M. R. Gaberdiel, "A non-supersymmetric open-string theory and S-duality," Nucl. Phys. B 499, 183 (1997) hep-th/9701137.

[24] J. A. Harvey, "String duality and non-supersymmetric strings," Phys. Rev. D 59, 026002 (1999) hep-th/9807213.

[25] S. Kachru and E. Silverstein, "Self-dual nonsupersymmetric type II string compactifications," JHEP9811, 001 (1998) hep-th/9808056.

[26] G. T. Horowitz and L. Susskind, "Bosonic M theory," hep-th/0012037.

[27] E. Witten, "Some computations in background independent off-shell string theory," Phys. Rev. D 47, 3405 (1993) hep-th/9210065; J. A. Harvey, D. Kutasov and E. J. Martinec, "On the relevance of tachyons," hep-th/0003101; D. Kutasov, M. Marino and G. Moore, "Some exact results on tachyon condensation in string field theory," JHEP0010, 045 (2000) hep-th/0009148; A. A. Gerasimov and S. L. Shatashvili, "On exact tachyon potential in open string field theory," JHEP0010, 034 (2000) hepth/0009103; S. L. Shatashvili, "On the problems with background independence in string theory," hep-th/9311177; S. L. Shatashvili, "Comment on the background independent open string theory," Phys. Lett. B 311, 83 (1993) hep-th/9303143. 
[28] F. Dowker, J. P. Gauntlett, G. W. Gibbons and G. T. Horowitz, "The Decay of magnetic fields in Kaluza-Klein theory," Phys. Rev. D 52, 6929 (1995) hep-th/9507143.

[29] M. S. Costa and M. Gutperle, "The Kaluza-Klein Melvin solution in M-theory," hepth/0012072.

[30] J. Maldacena, J. Michelson and A. Strominger, "Anti-de Sitter fragmentation," JHEP9902, 011 (1999) hep-th/9812073.

[31] M. Bershadsky, Z. Kakushadze and C. Vafa, "String expansion as large N expansion of gauge theories," Nucl. Phys. B 523, 59 (1998) hep-th/9803076.

[32] N. Nekrasov and S. Shatashvili, "On Non-Supersymmetric CFT in Four Dimensions", Phys. Rept. 320, 127 (1999); E. Silverstein, unpublished.

[33] C. Vafa, "Quantum Symmetries Of String Vacua," Mod. Phys. Lett. A 4 (1989) 1615.

[34] M. R. Garousi, "String scattering from D-branes in type 0 theories," Nucl. Phys. B 550, 225 (1999) hep-th/9901085.

[35] O. Aharony, M. Berkooz, and E. Silverstein, work in progress.

[36] P. Breitenlohner and D. Z. Freedman, "Positive Energy In Anti-De Sitter Backgrounds And Gauged Extended Supergravity," Phys. Lett. B 115, 197 (1982).

[37] J. Maldacena and H. Ooguri, "Strings in $\operatorname{AdS}(3)$ and SL(2,R) WZW model. I," hepth/0001053.

[38] D. Kutasov and N. Seiberg, "Number of degrees of freedom, density of states and tachyons in string theory and CFT," Nucl. Phys. B 358, 600 (1991).

[39] M. R. Douglas and B. Fiol, "D-branes and discrete torsion. II," hep-th/9903031. M. R. Douglas, "D-branes and discrete torsion," hep-th/9807235.

[40] D. Berenstein and R. G. Leigh, "Discrete torsion, AdS/CFT and duality," JHEP0001, 038 (2000) hep-th/0001055.

[41] C. Csaki, W. Skiba, and J. Terning, "Beta Functions of Orbifold Theories and the Hierarchy Problem", Phys. Rev. D 61, 025019 (2000); P. H. Frampton and W. F. Shively, "Conformal N=0 d=4 Gauge Theories from AdS/CFT Superstring Duality?", Phys. Lett. B 45449 (1999). 